\title{
10-Gb/s Slow-light Performance Based on SBS Effect in Optical Fiber using NRZ and PSBT Modulation Formats
}

Lilin Yi (1,2), Yves Jaouën (1), Renaud Gabet (1), Weisheng Hu (2), Yikai Su (2), Sébastien Bigo (3)

1: GET/Telecom Paris, CNRS UMR5141, 46 rue Barrault, 75634 Paris, France. Email: lilin.yi@enst.fr

2: SKL of AOCSN, Shanghai Jiao Tong University, 800 Dongchuan Rd, Shanghai 200240, China

3: Alcatel-Lucent France Research \& Innovation, Route de Villejust, 91620 Nozay, France

\begin{abstract}
For the first time, we have demonstrated error-free operation of slow-light via SBS in optical fiber for $10-\mathrm{Gb} / \mathrm{s}$ NRZ and PSBT formats. The PSBT signal sensivity is 5.2dB better than the NRZ case for a 35-ps delay.
\end{abstract}

\section{Introduction}

Recently, slow-light has attracted much interest due to potential applications in future optical networks, such as optical buffering and tunable time delay. Among all proposed techniques, slow-light mechanism based on Stimulated Brillouin Scattering (SBS) in optical fibre has attracted extensive attention [1-4]. The problem of narrow-band SBS gain has been solved by direct modulation of a pump laser diode using a Gaussian noise source [2]. Until now, the system performance for $10-\mathrm{Gb} / \mathrm{s}$ signal transmitted in SBS based slow-light have been investigated only in case of differential-phase-shiftedkeying (DPSK) modulation format [3]. However, the sensitivity was degraded by $\sim 10 \mathrm{~dB}$ for a $42-p s$ delaytime due to SBS filtering effect and chromatic dispersion accompanied with slow-light.

In this paper, we investigate the system performance of 10-Gb/s non-return-to-zero (NRZ) and phaseshaped binary transmission (PSBT) modulation formats in SBS based slow-light delay line. It is well-known that PSBT format allows high spectral efficiency and strong dispersion-tolerance [5]. Here, we propose to utilize the PSBT format to reduce the signal distortions in slow-light. All the results in term of sensitivity and power penalty have been measured at a bit-error-rate (BER) of $10^{-9}$. For the $10-\mathrm{Gb} / \mathrm{s}$ PSBT signal, a negative power penalty, i.e. $-2 \mathrm{~dB}$ for a 25 ps delay-time, has been obtained. When the delay-time is increased to $35 \mathrm{ps}$, the sensitivity of the PSBT signal is $5.2 \mathrm{~dB}$ better than the NRZ signal case. Maximum of $\sim 50$ ps delay with error-free operation can be obtained only by using PSBT format.

\section{Experimental setup}

Fig.1 depicts the experimental set-up. The signal transmitter consists of a laser diode (LD1) operating at $1548.26 \mathrm{~nm}$, and a Mach-Zehnder modulator (MZM) driven by a 10-Gb/s pseudo-random bit sequence (PRBS). A 10-Gb/s PBST modulation format can be achieved by filtering the electrical NRZ signal using a $5^{\text {th }}$ order Bessel filter with a $\sim 2.7 \mathrm{GHz}$ cut-off frequency [6]. A $2^{7}-1$ word length sequence is used for BER measurements due to non-optimized $3-\mathrm{GHz}$ PSBT electrical filter and the driver [7]. The inset of Fig.1 corresponds to the eye diagram of the PSBT signal. The signal is launched into a $20-\mathrm{km}$ long Truewave (TW) fibre with a $-10.75 \mathrm{GHz}$ Brillouin frequency shift. The SBS pump source is a directly modulated laser diode (LD2), whose central wavelength can be precisely controlled by temperature. The pump LD is modulated by a Gaussian noise source (Tektronix AFG3252), and subsequently boosted by a high power erbium-doped fibre amplifier (EDFA). We have obtained $\sim 12 \mathrm{GHz}$ SBS pump bandwidth, resulting in $\sim 7 \mathrm{GHz}$ gain bandwidth for a 22-dBm pump power.

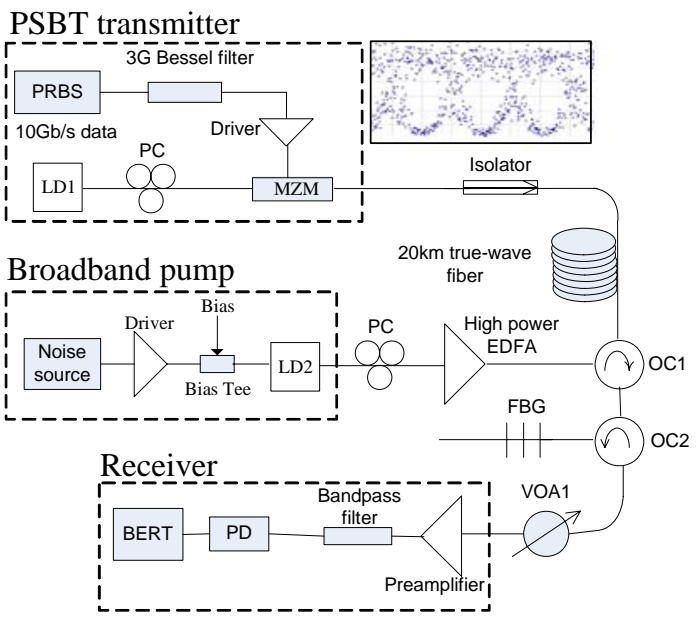

Fig.1 Experimental set-up. (LD) laser diode, (MZM) MachZehnder modulator, (EDFA) Erbium-doped fibre amplifier, $(P C)$ polarization controller, (OC) optical circulator, (FBG) fiber Bragg grating, (VOA) variable optical attenuator, (PD) photodiode, (BERT) bit-error-rate tester.

The coherent crosstalk between the signal and Rayleigh backscattering of the broadband pump is the dominant noise contribution in 10-Gb/s SBS based slow-light. The input signal power is set to $5 \mathrm{dBm}$, thus the Rayleigh backscattered power of the broadband pump is $\sim 20 \mathrm{~dB}$ lower than the output signal power, which still induces coherent crosstalk. Therefore we used a $\sim 0.1 \mathrm{~nm}$ bandwidth fibre Bragg grating (FBG) to minimize the crosstalk. The receiver consists of an optical preamplifier, a tunable optical filter, a 10-Gb/s PIN-FET photodetector (PD) and a bit-error-rate tester (BERT). Before the receiver, a variable optical attenuator (VOA) is used in order to tune the optical power for the BER measurement. For comparison, we have measured the delay performances of both the NRZ and PSBT formats. The NRZ modulation format is simply obtained by removing the Bessel filter, and optimizing the bias of the MZM and the electrical driven voltage. 


\section{Results and discussions}

Fig.2 shows the delay, eye diagram and BER evolutions of the $10-\mathrm{Gb} / \mathrm{s} \mathrm{NRZ}$ signal for different pump power levels. The larger the pump power, the higher the gain value and the delay-time are. The delay-times are 17, 35 and 50 ps for 19, 21 and 22$\mathrm{dBm}$ pump powers, respectively. However, the gain bandwidth is reduced when the pump power enhances because the Brillouin gain exponentially increases with the pump power. A narrow gain bandwidth induces strong filtering effect, which is the main cause of the signal distortion in all the previous SBS based slow-light demonstrations. When the pump power is increased to $22 \mathrm{dBm}$, error-free operation cannot be obtained even though we have detuned the carrier from the gain peak to suppress the filtering effect [3-4]. The maximal delay-time for $\mathrm{BER}<10^{-9}$ is $35 \mathrm{ps}$.
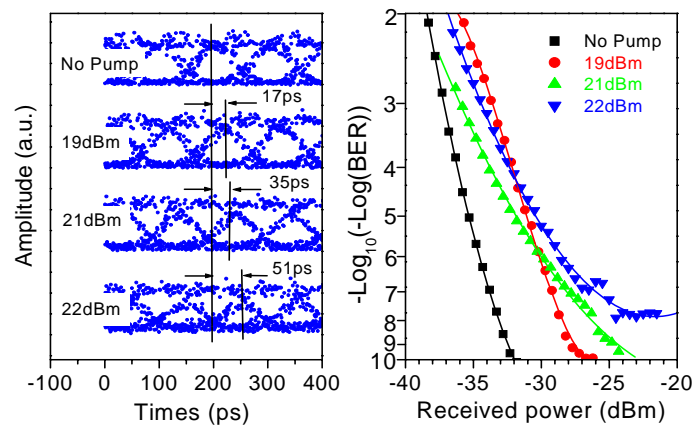

Fig.2 Delay-time, eye diagram and BER evolutions with pump power for 10-Gb/s NRZ signal.
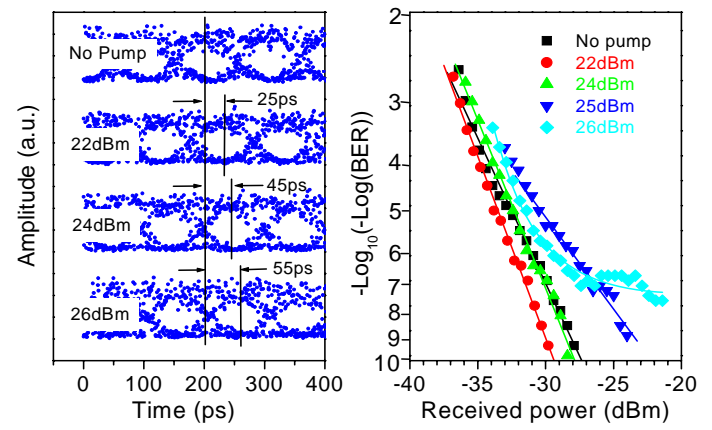

Fig.3 Delay-time, eye diagram and BER evolutions with pump power for 10-Gb/s PSBT signal.

The results are different for the 10-Gb/s PSBT format. Fig.3 shows the delay, eye diagram and BER evolutions of $10-\mathrm{Gb} / \mathrm{s}$ PSBT signal for different pump power levels. Without the pump, the receiver sensitivity is $-28.2 \mathrm{dBm}$, which has taken into account the FBG filtering effect. The SBS mechanism acts as a narrow-band filter, which suppresses the small ripples and increases the eye opening of the "O" bits, so the signal quality is improved after the SBS amplification and the delay. The high-frequency ripples mainly consist of $10-\mathrm{GHz}$ sinusoidal components [6], which can be filtered by the narrow band SBS gain. From Fig.3, when the pump power is set to $22 \mathrm{dBm}$ corresponding to $7-\mathrm{GHz}$ gain bandwidth and 5.6-dB On-Off gain, the delay-time is
$25 \mathrm{ps}$, and the eye opening is improved and the sensitivity achieves an optimum of $-30.2 \mathrm{dBm}$. In addition to the filtering effect, the improved sensitivity is also attributed to the dispersion-tolerance of the PSBT format, which can reduce the dispersiondistortion induced by the slow-light effect. However, if we further increase the pump power, the narrower SBS gain filters the high frequency components of the PSBT signal, which induces the signal distortion and sensitivity degradation. When the pump power is increased to $26 \mathrm{dBm}$, corresponding to an 11.2-dB On-Off gain, the delay-time is increased to 55 ps but error-free operation cannot be achieved.

Finally, we measured the time delay and the sensitivity variation with the SBS On-Off gain for the 10-Gb/s NRZ and PSBT signals, shown in Fig. 4. For the NRZ and the PSBT signals, the delay is linearly increased with the gain, and the maximum delay-times with error-free operation are 35 ps and 50 ps, respectively. For the NRZ signal, the sensitivity becomes worse with the increase of the delay. However, the sensitivity of the PSBT signal firstly improves to an optimum value and then degrades with the delay. For the same amount of 35-ps delay, the sensitivity of the PSBT is 5-dB better than the NRZ case. Moreover, the performance of the PSBT format could be improved using an optimized electrical PSBT filter [8].
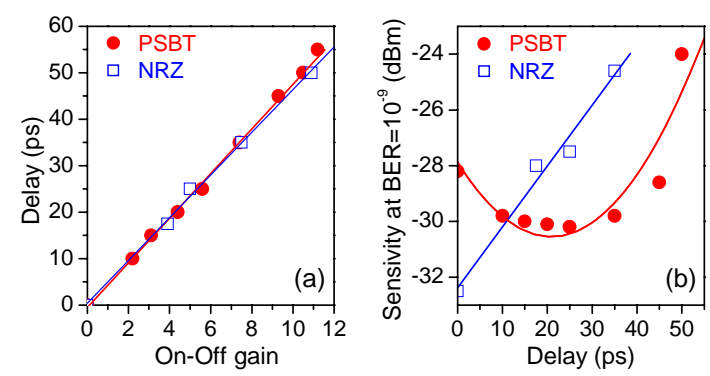

Fig. 4 (a) Delay versus the signal gain and (b) sensitivity versus the delay for 10-Gb/s NRZ and PSBT signals.

\section{Conclusions}

By using PSBT modulation format which allows high spectral efficiency and strong dispersion-tolerance, the system performance of a SBS based slow-light delay line is improved compared with the NRZ case. A negative power penalty for $B E R=10^{-9}$ has been obtained after delay (i.e. $-2 \mathrm{~dB}$ for 25 ps delay-time). Error-free operation can be achieved for a delay-time up to 50 ps only with the $10-\mathrm{Gb} / \mathrm{s}$ PSBT format, which is better than any previously reported results.

\section{References}

1 K. Y. Song, et al, Opt. Express 13(2005), page 82

2 Z. Zhu, et al, OFC 2006, paper PDP1

3 B. Zhang, et al, OFC 2007, paper OThD2

4 Lilin Yi, et al, IEEE PTL 19(2007), page 619

5 D. Penninckx, et al, IEEE PTL 9(1997), page 259

6 H. Kim, et al, IEEE PTL 14(2002), page 120

7 T. Franz et al. ECOC 1997, paper $n^{\circ} 448$, page 67

8 G. Charlet, et al. ECOC 2002, paper PD4.1. 